\title{
The Analysis for the Affecting Factors of Earth Synchronous Satellite Orbit Determination Error
}

\author{
Huifen $\mathrm{Li}$ \\ Joint Lab of Flight Vehicle Ocean-based Measurement \\ and Control \\ CSMTC \\ Jiangyin, China \\ kj1906@sina.com \\ Zhengxu Dai \\ Joint Lab of Flight Vehicle Ocean-based Measurement \\ and Control \\ CSMTC \\ Jiangyin, China \\ kj1903@sina.com
}

\author{
Jinbiao Zhou \\ Joint Lab of Flight Vehicle Ocean-based Measurement \\ and Control \\ CSMTC \\ Jiangyin, China \\ kj1121@sina.com \\ You $\mathrm{Wu}$ \\ College of Science \\ Hohai University \\ Naijing, China \\ wylhf@yeah.net
}

\begin{abstract}
Aiming at the preliminary orbit determination error of certain shipboard microwave unified tracking telemetry and command system of $\mathrm{C}$ frequency band(UCB) equipment in performing the earth synchronous satellite laughing missions, by analyzing correction, axes- systematic error propagation and instrumentation ship error track, focusing on the problems sorted. Analyses the methods of error tracing, orbit determination based on analyzing the effects of constant error, axes-systematic error and ship's position error. The problem of a big UCB injection error is a consequence of improper use of orbit model and inaccurate calibration of optical-electrical axis non-parallelism, which is caused by the change of optical axis. Accurate positioning problems and the effective methods is provided for orbit determination strategy and optical axis changes.
\end{abstract}

Keywords-orbit determination error; orbit elements; earth synchronous satellite; axes-systematic error; preliminary orbit determination

\section{INTRODUCTION}

It was found certain TT\&C equipment has a large injection error in certain earth synchronous satellite laughing mission, which is outdistance preliminary orbit determination accurate of the same type mis-sions' instrumentation ship equipments, already can' $t$ meet the requirements for the general depart-ment. It is found that the injection is too large by sta-tistics the same equipment in same type mission. So it's necessary to analyzing the status and parameters for solving the problems.

\section{PROBLEM ANALYSIS}

The orbit calculation need following data: shipboard equipments tracking data; ship swaying, deformation and position data. The ship swaying, deformation and ship position data is needed in all the shipboard equipments orbit determination calculation. Because the other equipments' orbit determination precision meets requirements, the working for troubleshoot problems is aimed at testing data of UCB equipment itself.

In the satellite laughing mission, the radar measuring data calculated parameters at injection got a high precision which used same antenna with UCB equipment, the consistency is better than the precise orbit element. Compared with the precise orbit element, the error of semi-major axis, which calculated by the UCB data, is seventy times than the radar.

The radar tracking time slot is prior to the separate of the rocket and satellite, but the UCB equipment is after the separation. It's also need to pay attention if the target characteristics have any change. Besides, two equipments use the same antenna, except the difference of opticalelectrical axis non-parallelism and $\mathrm{Cs}, \mathrm{Ce}$, the axessystematic error use the same parameters[1]. The calibration and use cases of optical-electrical axis nonparallelism should be the main emphasis in troubleshooting.

\section{ANALYSIS OF THE ORBIT DETERMINATION METHODS EFFECTS}

From the data source of orbit determination, radar orbit determination use the data 80 seconds before the separation of the rocket and satellite, UCB data orbit determination use the data 150 seconds after the separation of the rocket and satellite.

In consideration for a longer time span and a further away from the separation time of the rocket and satellite of using the orbit determination of the separation of the rocket and satellite, so trying to calculate orbit determination in PUVM2 method (orbit determination method of revise perturbation)[2]. It is found that PUVM2 orbit determination precision is better than UVM1 (orbit determination method of non-revise perturbation)[3]. 
The two calculating methods obtain dozens of kilometers from the results of the semi-major axis, and the result of PUVM2 is much more close to orbit determination. PUVM2 is better than UVM1 for the earth synchronous satellite laugh missions. The earth synchronous satellite orbit eccentricity is large (larger than
0.73), revise perturbation brings obvious effect for semimajor axis[4].

In the period of 80 seconds before, 134 seconds after of the separation of rocket and satellite, if not revise the perturbation, the semi-major axis are shown in Fig .1; if revise the perturbation, Fig .2 shows the semi-major axis change beyond requirements of General Department.

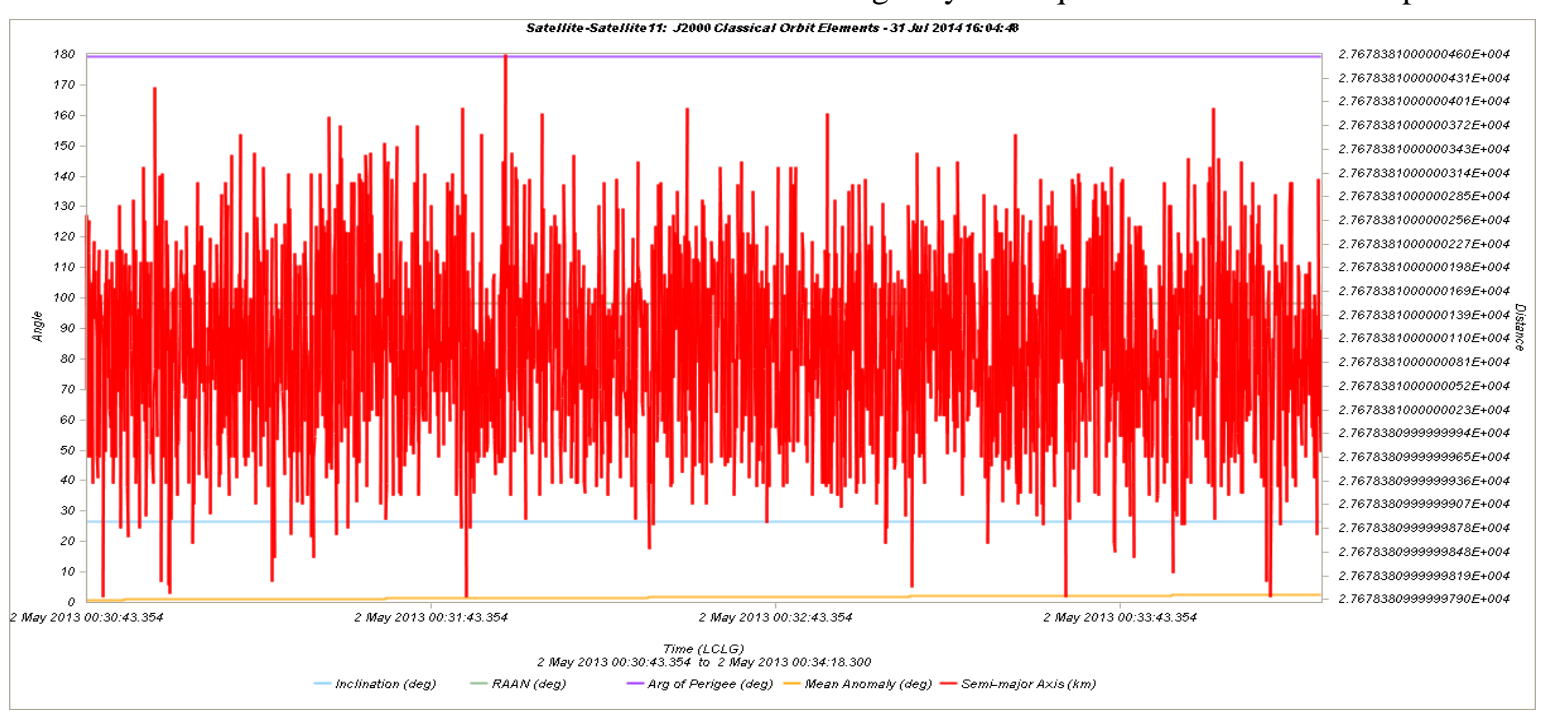

Figure 1. The result of UVM1 (large eccentricity orbit,the delta equals semi-major axis difference of orbit determination result and precise orbit)

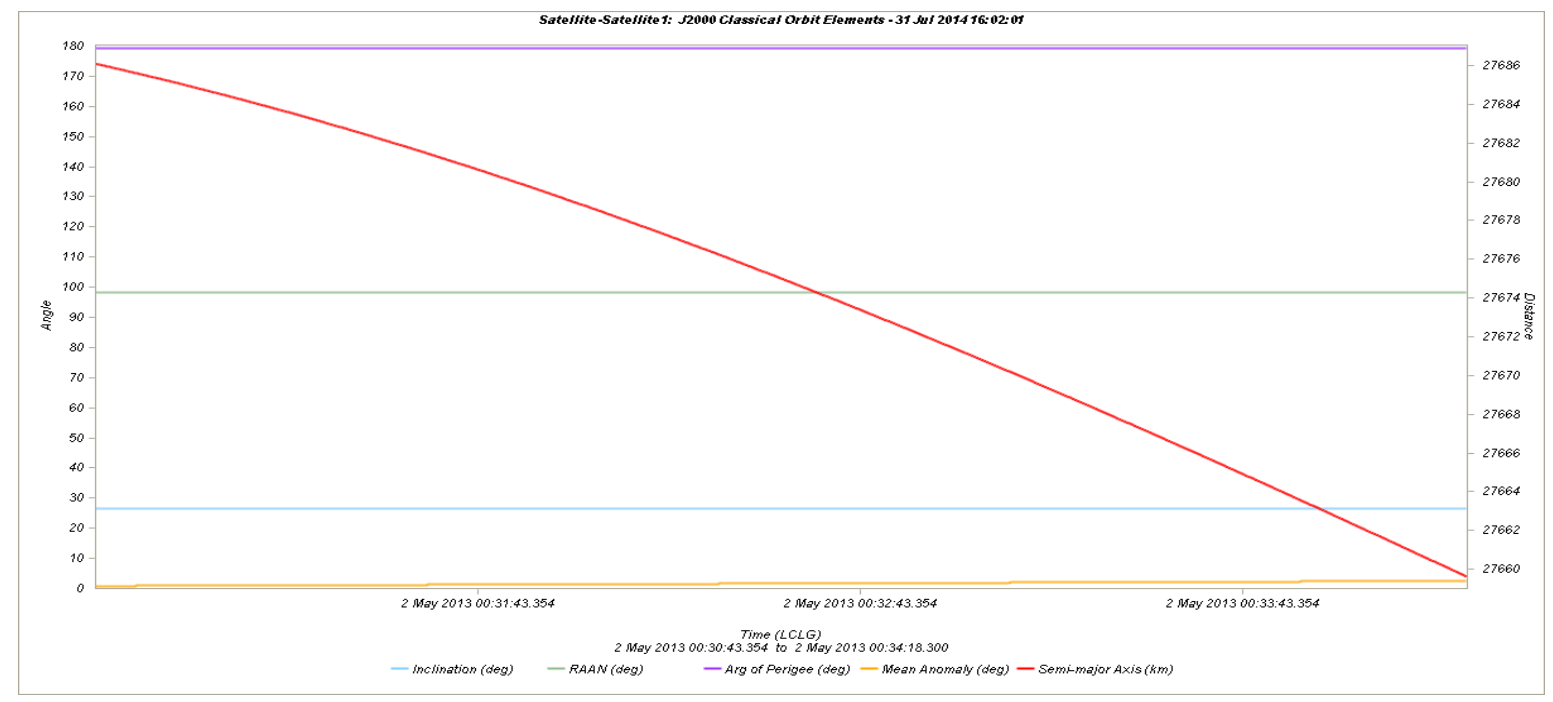

Figure 2. The result of PUVM2 (large eccentricity orbit,the delta equals semi-major axis difference of orbit determination result and precise orbit) 
Compared with the data before the separation of rocket and satellite, the data after the separation of rocket and satellite is a longer time span, and brings greater impact in ignoring perturbation.

\section{ANALYSIS OF DIFFICULT POINT FOR TROUBLESHOOTING}

The commonly used two methods for troubleshoot-ing, one is calculating the equipments measured value in using precise orbit element, and make a comparison in inertial horizontal coordinate system; the other one is based on the GPS data from the rocket, change the data from WGS-84 coordinate system to inertial horizontal coordinate system, then compare with instrumentation ship's data in inertial horizontal coordinate system.

\section{A. Feasibility analysis for method 1}

Using precise orbit element to calculate measured value, and compare with instrumentation ship's data, it is found that the data consistency is poorer. The analysis found that.

Large effects for orbit element precision

Analyzing the effects of orbit element precision to inertial horizontal coordinate system data, it is found precision of the precise orbit element at least should be: a contains up to 4 digits after the decimal point $(\mathrm{km})$, e contains up to 6 digits after the decimal point, i、 $\Omega$ 、 $\omega$ 、 $M$ contains up to 6 digits after the decimal point $\left({ }^{\circ}\right)$, part of the data contains up to 3 digits after the decimal point. The precision of the precise orbit element cannot meet the requirements.

Data precision of orbit calculates is inconformity

By understanding that the principle of precise or-bit calculates by TT\&C center is: preferred to use GPS data, the precision of orbit element is equal to the GPS data precision at this moment; mix data to-gether from each instrumentation station and calcu-lation when there is no GPS data; exclusive use in-strumentation ship's data when only contain the instrumentation ship's data, and the precision of or-bit element is just the precision of the shipboard equipments [5].

The introduction of error in orbit extrapolation

By using extrapolation in two processes when calculating accurate orbit element.

Process of orbit calculate. Calculate a group of orbit element forms a piece of observation data, and then calculate to the specific time.

Based on the accurate orbit element to calculate inertial horizontal coordinate system data, then extrapolate to the period of time which is needed to compare. Long-time extrapolation is bound to cause error[4].

Based on the above analysis, the situation will be complex by using accurate orbit element to analyze the problem, and can cause differences in compara-tive result, not suitable as a reference.

\section{B. Feasibility analysis for method 2}

The rocket is the tracking target of radar and USB; it could directly compare with on-board rockets' GPS data. The satellite is tracking target of UCB, the de-viation must be exist in comparison of rocket board GPS data. Must analyzing the angle deviation value of rocket and satellite after the separation.
By using the rocket board GPS data to calculate orbit element before the separation and revising fixed delta V, obtains the orbit element of rocket and satellite after their separation. It will get a clear un-derstanding of the changing situation from separa-tion to 134 seconds after the separation by calculat-ing the angle in orbit element extrapolation. So, by using rocket board data could analyze UCB meas-urement precision qualitative.

\section{MEASURING DATA ERROR ANALYSIS}

Comparing UCB measuring data after satellite-rocket separation with on-board GPS data, the result shows that the UCB data does not have an obvious distance error, while it has a big angular error which is far beyond the equipment's precision requirement.

Radar angle measuring data is consistent with GPS data, while it is not consistent with UCB angle measuring data. Except the optical-electrical axis non-parallelism $\mathrm{Cs} 、 \mathrm{Ce}$, the other data are corrected by the same series of correct parameters. Dealing with this mission's data and comparing the radar angle measuring data with GPS data, it satisfies equipment's precision requirement. As the instrumentation ship has just finished the calibration at the dock, based on the radar's data comparison result, the possibility of faults in axes-systematic error calibration can be eliminated.

UCB and radar use the same axes-systematic error parameters, while they use different optical-electrical axis non-parallelism parameters to correct angle measuring error. If in one same mission the angle measuring error of radar is small while that of UCB is large, we should focus on analyzing the accuracy of UCB optical-electrical axis non-parallelism.

In this mission, the optical-electrical axis nonparallelism parameters $\mathrm{Cs}$ and $\mathrm{Ce}$, which are used by UCB, are calculated with the beacon balloon data. Using the beacon balloon data to calculate $\mathrm{Cs}$ and $\mathrm{Ce}$ and comparing them with the $\mathrm{Cs}$ and $\mathrm{Ce}$ which are offered by the equipment operators, we find they are consistent. It shows that the calculating method and the use of beacon balloon data are correct.

Analyzing the condition of UCB on other instrumentation ship performing the same type of mission, we found that UCB on other instrumentation ship had a higher orbit determination precision. Our ship's UCB orbit determination precision was fine in one mission, while its injection errors were quite big in other missions. We discover that other ship's UCB use the parameters Cs and Ce which are calibrated at the dock, and the same as our ship's UCB when it obtained the high orbit determination precision.

The analysis above shows that the parameters Cs and Ce calculated with beacon balloon data are consistent with that calculated by equipment operators, and the calculating method and the use of beacon balloon data are correct. However, why using the parameters $\mathrm{Cs}$ and Ce calculated with the beacon balloon data is less effective than using Cs and $\mathrm{Ce}$ which are calibrated at the dock?

The axes-systematic error of ship-borne equipment is calibrated by using the telescope on the antenna aiming at the azimuth calibrator. The axis of telescope is called optical axis, and it represents the mechanical axis in calibration. Assuming the telescope's optical axis and 
mechanical axis are completely superposed, when the optical axis aims at one azimuth calibrator, the mechanical axis will aim at it too[6]. As a matter of fact, the telescope is not installed through the center of mechanical axis, meanwhile the optical axis and the mechanical axis are not completely paralleled. As a result, the optical-mechanical axis non-parallelism exists in the measuring mission[7].

Now a Low Light Level TV is installed on the radar to conduct calibration instead of the telescope.

When the radar tracks the object, the electrical axis points at the object, and the mechanical axis outputs the angle measuring data, which means the sensor outputs the mechanical axis' position.

Wireless measuring equipments track objects with the electrical axis of antenna, thus the direction of the electrical axis is objects' position. Therefore, we should zero-correct the measuring data to obtain the direction of the mechanical axis, then correct the optical-mechanical axis non-parallelism to obtain the direction of the optical axis, and correct the optical-electrical axis non-parallelism to find the direction of the electrical axis [8].

As we use angular Zero Value, optical-mechanical axis non-parallelism and optical-electrical axis non-parallelism during the correction, the precision of data depends on the veracity of these parameters' calibration. The angular Zero Value and the optical-mechanical axis non-parallelism are calibrated at dock, while the optical-electrical axis nonparallelism $\mathrm{Cs}$ and $\mathrm{Ce}$ are calibrated with beacon balloon before the mission[9]. Considering from the check result of Cs and Ce, the calculating method is correct, and the analysis above excluded the possibility of inaccuracy of the calibration of axes-systematic error at the dock. Therefore we speculate that the change of the optical axis of UCB calibration TV leads to the consequence that the optical-electrical axis non-parallelism, which is calibrated with the benchmark of optical axis, did not accurately reflect the real status of the equipment.

\section{PROBLEM LOCATION AND VERIFICATION}

As UCB and radar use the same antenna, the dynamic lag error of UCB minus that of radar is the orientation difference between antenna electrical axis of the two equipments.

Before satellite-rocket separation, radar is the main measurement equipment. Although UCB does not do tracking and surveying at that moment, it records effective angle error voltage data, which represents the orientation difference between the object and the electrical axis of antenna. Thus, we can obtain the orientation difference between antenna electrical axis of UCB and radar. The difference plus the optical-electrical axis non-parallelism of radar is the optical-electrical axis non-parallelism of $\mathrm{UCB}[10]$.

Data accumulating and analysis shows that radar's electrical axis of antenna is very stable, and $\mathrm{Cs}$ and $\mathrm{Ce}$ calibrated at the dock are adopted all previous. If we obtain the orientation difference between UCB and radar's antenna electrical axis, we can use radar's $\mathrm{Cs}$ and $\mathrm{Ce}$ to calculate UCB's Cs and Ce.

In order to verify the analysis above, basing on radar electrical axis of antenna, we use the effective angular error voltage recorded by UCB to calculate UCB opticalelectrical axis non-parallelism. Using the new optical- electrical axis non-parallelism to correct the data, the orbit determination precision trebled. However, comparing with radar data processing result, semi-major axis error is still large.

The model error influence analysis shows that whether correcting perturbation (PUVM2 or UVM1) would bring an influence of decades of kilometers long on semi-major axis in the injection period of earth synchronous satellite orbit determination mission. The error is still big after correcting the data with the new optical-electrical axis non-parallelism, and we assume that it is course by the model error. To verify this assumption, we use the new optical-electrical axis non-parallelism to process UCB data and then use PUVM2 to determinate orbit. The result shows in table 1 that the orbit determination precision can satisfy ship-board equipment's requirement.

TABLE I. RESULT OF ORBIT DETERMINATION

\begin{tabular}{|c|r|c|}
\hline Tasl & $\Delta \mathrm{a}(\mathrm{km})$ & Method of orbit determination \\
\hline \multirow{2}{*}{ A } & -33.341 & Uvm1+calibration of optical-electrical axis non-par all elism \\
\cline { 2 - 4 } & 6.764 & Puvm2+new optical-electrical axis non-parallelism \\
\hline \multirow{2}{*}{ B } & -41.446 & Uvm1+calibration of optical-electrical axis non-par all elism \\
\cline { 2 - 4 } & 6.161 & Puvm2+new optical-electrical axis non-parallelism \\
\hline \multirow{2}{*}{ C } & -32.658 & Uvm1+calibration of optical-electrical axis non-par all elism \\
\cline { 2 - 4 } & 9.633 & Purm2+new optical-electrical axis non-parallelism \\
\hline \multirow{2}{*}{ D } & -74.356 & Uvm1+calibration of optical-electrical axis non-parallelism \\
\cline { 2 - 4 } & -12.693 & Puvm2+new optical-electrical axis non-parallelism \\
\hline \multirow{2}{*}{ E } & -17.049 & Uvm1+calibration of optical-electrical axis non-parall elism \\
\cline { 2 - 4 } & 11.578 & Puvm2+new optical-electrical axis non-parallelism \\
\hline
\end{tabular}

Thus, we can confirm that the problem of a big UCB injection error is a consequence of improper use of orbit model and inaccurate calibration of optical-electrical axis non-parallelism, which is caused by the change of optical axis.

\section{CONCLUSIONS}

Aiming at the problem of a big injection error during UCB performing earth synchronous satellite launch mission, this paper analyzed its mechanism and took some troubleshooting measures. Based on analyzing the influence of instrumentation ship measuring er-ror, this paper made qualitative analysis and quanti-tative analysis of error tracing, located the problem accurately in opticalelectrical axis non-parallelism and orbit determination method, and advanced effec-tive measures to solve this problem.

\section{REFERENCES}

[1] L.Huifen and Z.Zhonghua, "The exterior tracking \& measuring data analyzing and processing of instrumentation ship," Journal of Spacecraft TT\&C Technology,vol. 27, Dec. 2008, pp.65-70.

[2] M.Yongxing and N.Siaoqiu."Weighting Method of Orbit Determination for Multiple Orbit Measurement Data Fusion in Satellite Injection Phase," Telecommunication Engineering. Vol.55,No.9. Sep.2015. pp.993-999.

[3] M.Yongxing.Unit Vector Method of Spacecraft Orbit Determination. Beijing:National Defense Industry Press,2007. 3C

[4] Z.Yuxiang, Satellite Orbit Determination. Beijing: National Defense Industry Press. 2007.

[5] M.Yongxing and M.Jingyuan."An Orbit Determination Method Using Data Before and After Satallite-Rocket Separation,” Journal of Astronautics. Vol.35,No.12. Dec.2014. pp.1359-1366.

[6] J.Wenda, Space TT\&C Ship. Beijing: National Defense Industry Press. 2002. 
[7] Z. Dean, Technology of calibration and flight test for TT\&C ship's measuring-sommunicating equipment. Beijing: National Defense Industry Press. 2009.

[8] M.Nanping and W.Shenghui."Dynamic Calibration of NonParallelism between Ship-borne Radar's Optical Axis and Mechanical Axis," Telecommunication Engineering. Vol.54,No.9. Sep.2014. pp.1210-1215.
[9] L.Huifen, "Adaptive Processing of Abnormal Value in Measured Data by Space Tracking Ship ". Telecommunication Engineering. Vol.51,No.4. Apr.2011. pp.54-59.

[10] W.Ming and H.Panfeng."Identification of mass Characteristic Parameters for Non-cooperative Target Spacecraft,'FLIGHT DYNAMICS.Vol.32.No.6.Dec.2014.pp.536-540. 